\title{
The evolution of Jupiter family comets over 2000 years
}

\author{
G. TANCREDI and H. RICKMAN \\ Astronomiska Observatoriet, Box 515, S-75120 Uppsala, Sweden
}

\begin{abstract}
.
The orbital evolution of the whole sample sample of short-period comets was computed by numerical integrations for a time interval of $2000 \mathrm{yr}$ centered on the present epoch. This data base is intended to serve in various studies involving the statistics of orbital evolution and correlation with physical parameters or discovery circumstances. We present some results concerning the following aspects: the evolution of the orbital elements and their past-future asymmetry, statistics on the discovery of comets and on the encounters of comets with Jupiter.
\end{abstract}

Key words: short-period comets - dynamical evolution

\section{Introduction}

The numerical integration of the orbital evolution of short-period comets (hereafter $s p c$ ) is a powerful method to analyse the characteristics of this sample of objects. Due to inherent errors of numerical methods and to the chaotic behaviour of the majority of $s p c$ orbits, it is not possible to draw definitive conclusions about the particular evolution of any one object, but we can use these integrations to extract statistical information on a sample of objects that resembles the evolution of the observed population.

The rapid development of and easy access to fast computers has made the study of the whole population of $s p c$ feasible for any research group interested in the problem. Since the first set of integrations done by Carusi et al. (1985) in the frame of the Long Term Evolution Project (LTEP), several groups have performed similar work. Compared to the first project there have been some improvements in several aspects of the integrations, but the main reason to perform a new numerical integration of the observed sample of $s p c$ is the easy access to the data.

\section{Our integrations}

The numerical integrations were performed with the 15th order RADAU integrator with variable stepsize developed by Everhart (1985). Each comet was integrated separately with the 9 planets. Non-gravitational (hereafter $n g$ ) forces for the comets have been taken into account in case a value was stated in the Catalogue of Cometary Orbits, 6th Edition (hereafter CCO6, Marsden 1989). As the ng parameters are calculated by the linkage of observations during several apparitions of a comet and some of the linkages overlap, we decided to use a mean value of the different $n g$ parameters stated for a certain instant.

We chose as initial values for the planets the masses, heliocentric positions and velocities for JD 2448000.5 from JPL DE 118. The cometary elements were taken from the last orbit listed in the CCO6 plus later updates from the MPC. $163 \mathrm{spc}$ $(P<200 \mathrm{yr})$ were included: 143 of the Jupiter Family $(P<20 \mathrm{yr})$ (hereafter JF) and 20 of the Halley type $(20<P<200 \mathrm{yr})$.

We performed several sets of integrations to study different aspects of the dynamical evolution of spc. The main set of integrations, to which most of our results 
refer, corresponds to $\pm 1000 \mathrm{yr}$ starting at JD 2448000.5 (1990.3) with output every 500 days.

Due to round-off errors and the different stepsize evolution in each integration, the "planetary systems" used in the 163 separate integrations have slight differences that grow with time from the starting point of the integrations. For the terrestrial planets the standard deviations of position and velocity comparing the 163 integrations grow to the order of $10^{-9.5} \mathrm{AU}$ and $10^{-11} \mathrm{AU} /$ day, respectively, while for the outer planets the values are half and one order smaller, respectively.

Our aim is to reproduce the orbital evolution of spc during the observed period of time as well as possible. To check how well we achieved this objective, we compared the orbital elements obtained in our integrations to those appearing in the $\mathrm{CCO} 6$ at each perihelion passage. To compare the slowly evolving orbital elements $(a, e, i, \omega$ and $\Omega$ ) the D-criterion (Southworth and Hawkins 1963) was computed at each passage and we always got values less than $10^{-3}$. The difference in the time of perihelion passage $\left(\Delta T_{p}\right)$ was also computed and turned out to be $\left|\Delta T_{p}\right|<10^{-3}$ days.

Hereafter we will only present results concerning the dynamical evolution of $\mathrm{JF}$ comets. We regard the JF and Halley-type comets as representing two different populations of objects in the dynamical sense (Carusi and Valsecchi 1987) and hence they require separate analyses.

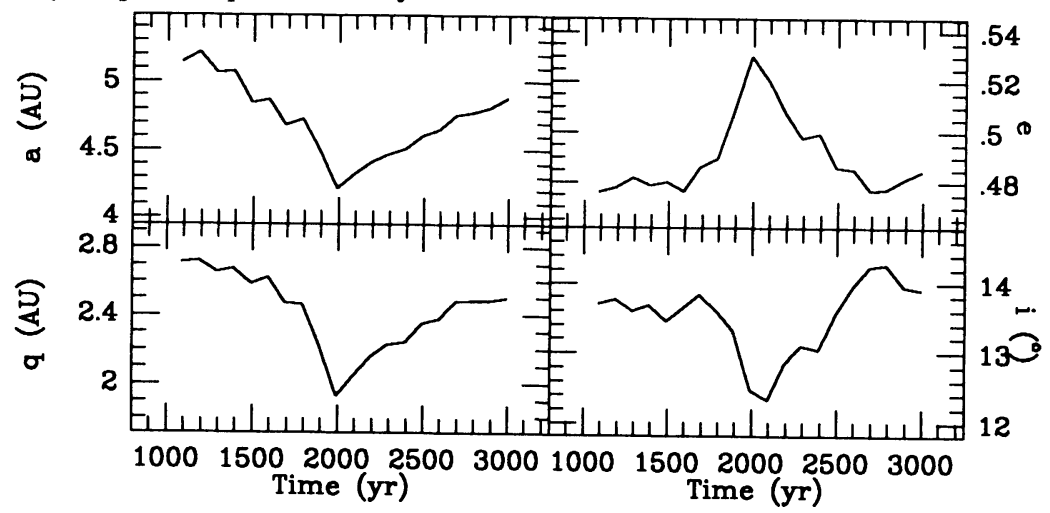

Fig. 1. Mean values of $a, q, e$ and $i$ for the JF comets as functions of time.

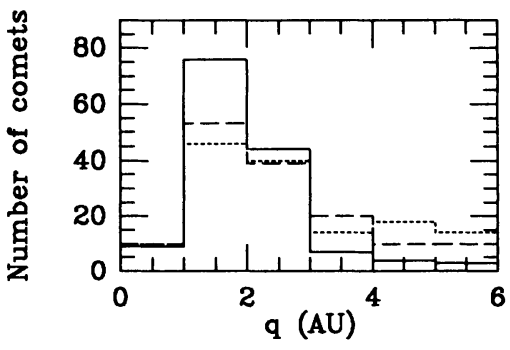

Fig. 2. Histogram of the mean cometary distribution of $q$ for three intervals of time : 1000-1100 (dotted line), 1950-2050 (full line) and 2900-3000 AD (dashed line).

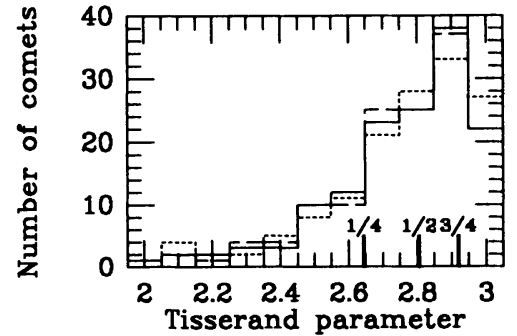

Fig. 3. Same as Fig. 2 for the Tisserand parameter $T$. Comets $\mathrm{P} /$ Tuttle and $\mathrm{P} / \mathrm{Machholz}$ lay outside the plotted range $(T<1.95)$. 


\section{General features}

Fernández (1985) (hereafter F85), based on results of the LTEP, observed a clear concentration of comets towards smaller perihelion distances at present compared to the distribution in the past and future. He explained this effect as an observational bias because comets tend to be discovered when they get low- $q$ orbits. We looked into the same problem by taking the mean of different orbital elements and plotting them as functions of time (Fig. 1). We observe that not only $q$ but all the plotted elements have extrema close to the present time. Obviously the evolutions of $a, e$ and $q$ are intimately related, but this is less evident for $i$. Although the dip for $i$ is not very pronounced, what strikes us is the fact that it almost coincides with the present epoch. The phenomenon can be explained at least partially as a selection effect due to the fact that one tends to search for comets at low latitudes. Figs. 2 and 3 show histograms of the mean cometary distribution of $q$ and $T$ (the Tisserand parameter), respectively, for three intervals of time : 1000-1100, 1950-2050 and 2900$3000 \mathrm{AD}$. The minimum of mean $q$ is seen to be due to a peaked distribution around 1-2 $\mathrm{AU}$ at the present time compared to the past and future. Although $T$ is not totally conserved during the evolution of a comet, mainly because Jupiter is not in a circular orbit, the general shape of its distribution is conserved, evidencing a clear concentration of $\mathrm{JF}$ comets at $T \approx 3$.
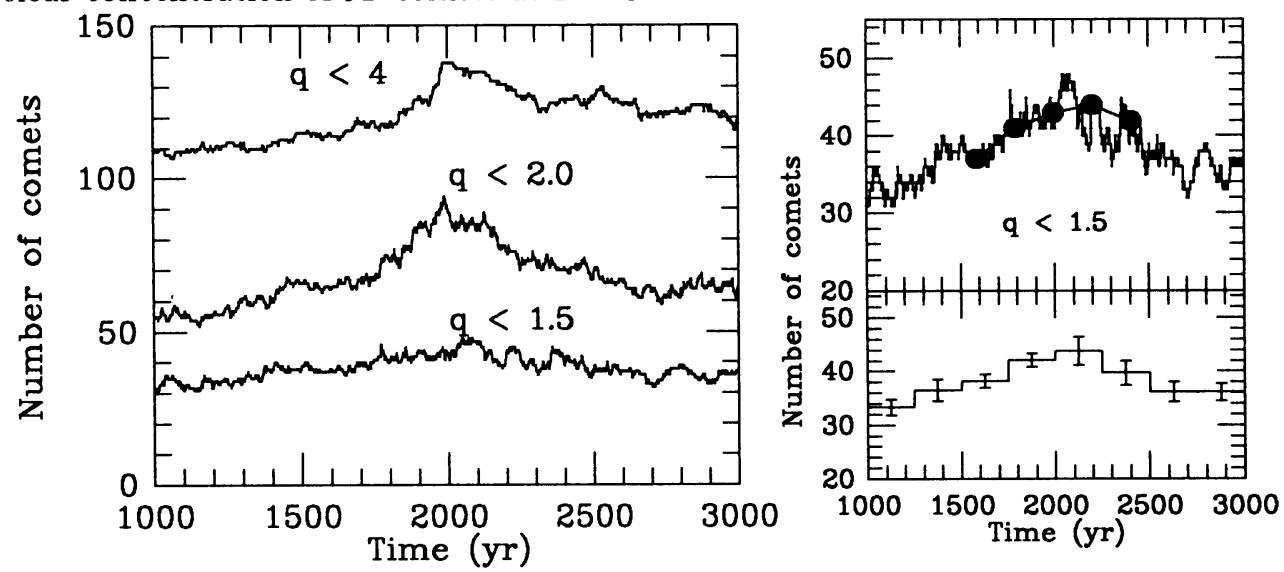

Fig. 4. a) Comets with $q$ less than certain limits as a function of time. b) Enhancement of Fig. $4 \mathrm{a}$ for $q<1.5 \mathrm{AU}$. The connected dots were obtained with the same sampling times as F85. c) Average number of comets with $q<1.5 \mathrm{AU}$ using a bin width of $250 \mathrm{yr}$.

\section{Lifetime of JF comets}

A past-future asymmetry in the cometary distribution of $a$ and $q$ (i.e. a larger number of comets with greater $a$ and $q$ in the past than in the future), pointed out by F85, is clearly seen in Fig. 1 as a higher value of the mean $a$ and $q$ in the past than in the future. Actually, one of the most interesting results obtained from the data of the LTEP is the estimate by F85 of the active lifetime of JF comets based on this past-future asymmetry in $q$. He plotted the number of comets with $q$ smaller than a certain limit for five different instants of time. We now repeat the same analysis to get a new estimate of the lifetime based on a larger sample and 
a longer integration. Fig. 4a shows the number of comets with $q$ less than certain limits as a function of time.

Although we do see a past-future asymmetry for the upper curves, this is hardly distinguishable for the curve of $q<1.5 \mathrm{AU}$, even with the enhancement presented in Fig 4 b. It is striking that if we plot our data for the exact sampling times of F85, the values for the future coincide with local maxima of the curve. In Fig. 4c we show the average number of comets with $q<1.5 \mathrm{AU}$ using a bin width of $250 \mathrm{yr}$ in order to filter out the statistical noise. The past-future asymmetry for this limit has disappeared at the $1 \sigma$-level. Only using extreme values for the corresponding F85 sample times (i.e. mean $-1 \sigma$ for the number of comets in the past and mean $+1 \sigma$ for the future) can we obtain similar results for the active lifetime (1000 revolutions for $g<1 \mathrm{AU}$ ). It thus seems that the latter can be viewed only as a lower limit to this important parameter, and that the independent estimate by Kresák (1981) is too low. Our data, unfortunately, does not allow to set any further limits.

\section{The discovery of comets}

As shown in Fig. 5, the discovery rate of JF comets has had a dramatic increase in the last 30-50 yr, mainly due to the discovery of comets with large $q$; in the beginning of this century the rate of discoveries with $q>1.5 \mathrm{AU}$ overtook that of smaller $q$. Except for the latest $20 \mathrm{yr}$, the discovery rate of comets with $q<1.5 \mathrm{AU}$ has been decreasing since the end of the last century. A closer look to the latest discoveries shows that many comets have $q$ close to the upper limit. From these facts it appears that we are reaching completeness of the sample of $s p c$ with $q \lesssim 1.5$ AU (Shoemaker and Wolfe 1982). With the available techniques it appears that we are also close to reaching completeness of the sample of observable large- $q$ comets. For $q>1.5 \mathrm{AU}, 92 \mathrm{JF}$ comets have been discovered. Assuming a population of a few thousand members (Fernández et al. 1991) and the magnitude distribution proposed by Hughes (1986), we find that the number of comets observable with the present techniques (absolute total mag. $H_{T} \lesssim 7$ ) is not much higher.

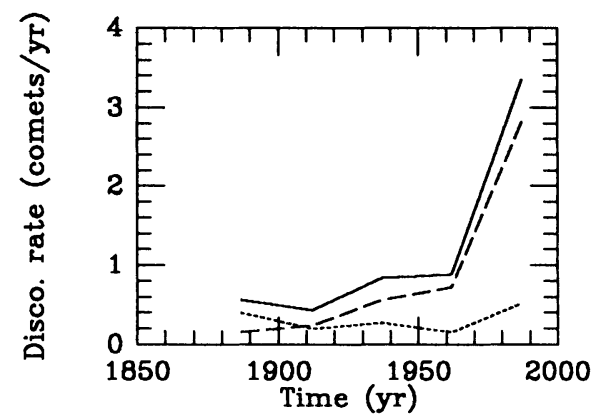

Fig. 5. The discovery rate of JF comets as a function of time (full line - total rate, dashed line - comets with $q>1.5 \mathrm{AU}$, dotted line - comets with $q<1.5 \mathrm{AU})$.

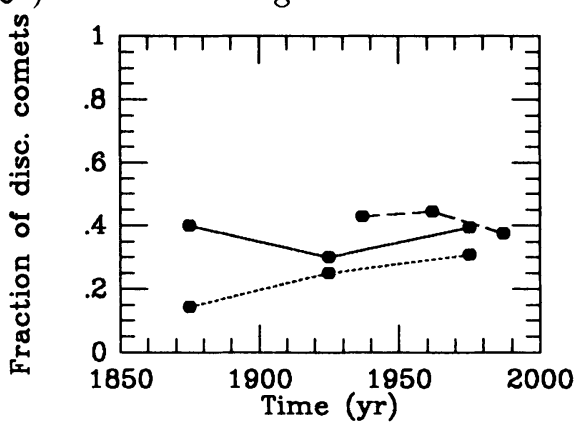

Fig. 6. Fraction of comets that has experienced a decrease in $q$ greater than 0.1 $\mathrm{AU}$ in the $20 \mathrm{yr}$ before discovery for different $q$ limits and bin width (see text).

A qualitative evolution with time is seen when we study the discovery statistics in relation to the preceding dynamical evolution. Karm and Rickman (1982) used a 
simple model to conclude that many comets have been discovered just after a close encounter with Jupiter, when their perihelion distance decreased. Fig. 6 shows the fraction of discovered comets that has experienced a decrease in $q$ greater than $0.1 \mathrm{AU}$ in the $20 \mathrm{yr}$ before discovery. The full line and dashed line correspond to discoveries of comets with $q>1.5 \mathrm{AU}$ and bin width of 50 and $25 \mathrm{yr}$ respectively; the dotted line to $q<1.5 \mathrm{AU}$ and bin width of $50 \mathrm{yr}$. The fractions always refer to the number of objects in each sample. Due to the statistical uncertainties, we can not see any particular trend for large $q$ comets. However, for comets with $q<1.5$ $\mathrm{AU}$, the fraction of comets that have jumped to lower $q$ prior to discovery in the present is twice that at the end of last century. Furthermore, the jumps observed in the last $40 \mathrm{yr}$ are much bigger than the ones observed in the previous years (from the 17 jumps with $\Delta q<-0.1 \mathrm{AU}, 4$ out of the 5 biggest jumps ocurred since 1950). Considering that we are reaching completeness of the population of "stable" orbits for this case we may explain the increase by the fact that we are approaching a state where we are left to discover only comets that have just entered the region.

\section{Encounters of comets with Jupiter}

We have already seen that comets tend to be discovered shortly after a close encounter with a respective decrease in the perihelion distance. We might ask if this phenomenon reflects a particular period of the evolution of the observed sample when they tend to have more encounters, or if the rate of encounters does not vary with time. In Fig. 7 we plot the evolution of the number of close encounters $(D<0.5$ AU to Jupiter) during the $2000 \mathrm{yr}$ integration. A peak starting at the present century and extending for a few centuries into the future is observed. The number of encounters at present is roughly twice that at the beginning of the interval.

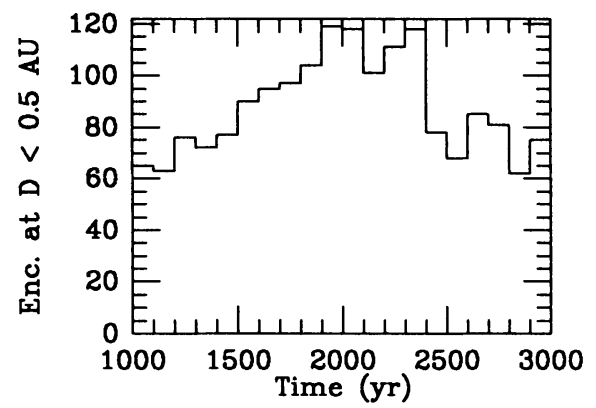

Fig. 7. Number of close encounters ( $D<0.5 \mathrm{AU}$ of the planet) during the $2000 \mathrm{yr}$ integration (bin width $100 \mathrm{yr}$ ).

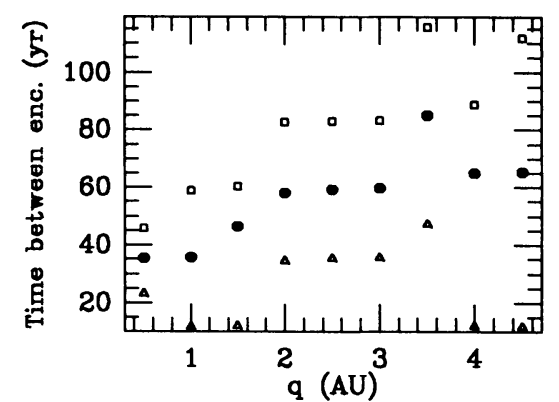

Fig. 8. Median (full dots), lower (triangles) and upper (squares) quartiles for the distribution of time between consecutive encounters for different $q$-intervals.

To explain this feature we would consider two possible alternatives. Comparing Figs. 1 and 7 , we see that the number of encounters tends to increase as the mean $q$ decreases. From our data set we obtain the frequency of encounters as a function of $q$. Fig. 8 shows the median, lower and upper quartiles for the distribution of time between consecutive encounters for different $q$-intervals. The increase seen is nearly the same as that of the mean orbital period with $q$. Comparing Figs. 2 and 
8 , we see that the shift of the $q$ distribution should lead to a peak of the encounter frequency at present, though by less than a factor two.

When a comet jumps to smaller $q$ (generally due to an encounter with Jupiter) it receives a greater insolation and the dust mantle formed at higher $q$ may be partially or totally blown off; consequently, the comet becomes brighter and the chance to discover it increases. Therefore, we tend to discover comets when their frequency of jumps (or equivalently frequency of encounters) is high. It is difficult to assess this picture quantitatevely, but at least qualitatively it agrees well with the observed distribution of encounters.

\section{References}

Carusi,A., Kresák,L., Perozzi,E., Valsecchi,G.B.: 1985, Long-Term Evolution of Short-Period Comets, Adam Hilger,Bristol,UK

Carusi,A. and Valsecchi,G.B.: 1987, in Z. Ceplecha and P. Pecina, ed(s)., Interplanetary Matter, Publ. Astron. Inst. Czechosl. Acad. Sci. No. 67, Vol. 2, 21

Everhart,E.: 1985, in A. Carusi and G.B. Valsecchi, ed(s)., Dynamics of comets: Their origin and evolution, D. Reidel Pub. Co., 185

Fernández,J.A.: 1985, 'Dynamical capture and physical decay of short-period comets', Icarus 64, 308

Fernández,J.A., Kámel,L. and Rickman,H.: 1991, in Fernández,J.A. and Rickman,H., ed(s)., International Workshop on Periodic Comets, Univerisdad de la Républica, Uruguay, submitted

Hughes,D.: 1986, in C.-I. Lagerkvist, B.A. Lindblad, H. Lundstedt and H. Rickman, ed(s)., Asteroids, Comets and Meteors II, Uppsala University, Sweden, 503

Karm,J. and Rickman,H.: 1982, 'Pre-discovery encounters between short-period comets and Jupiter estimated by a keplerian approximation', Bull. Astron. Inst. Czechosl. 33, 359

Kresák,L.: 1981, 'The lifetimes and disappearance of comets', Bull. Astron. Inst. Czechosl. 32, 321

Marsden,B.: 1989, Catalogue of Cometary Orbits, 6th Ed., Smith. Astroph. Obs., Cambridge, Massachussets

Shoemaker,E. M. and Wolfe,R.F.: 1982, in D. Morrison, ed(s)., Satellites of Jupiter, Univ. of Arizona Press, Tucson, Arizona, 277

Shouthworth,R.B. and Hawkins,G.S.: 1963, 'Statistics of meteor streams', Smithson. Contr. Astrophys. 7, 261

\section{Discussion}

G.B.Valsecchi - The overabundance of encounters with Jupiter observed close to the present epoch and in the near future should be tied to the minimum of the inclinations of comets that is also observed in the sample at about the same time, since the probability of planetary encounters goes as $1 / \sin i$.

G.Tancredi - After correcting the distribution of number of encounters by the change in the $q$-distribution, the small variation of mean $i$ (from 13.8 to 12.3, implying an increase of the probability of encounter by a factor of 1.12) could partially explain the remaining part of the peak. 